[Article]

\title{
多重网络增强的层状光子晶体水凝胶及应力变色响应
}

\author{
向双飞李 婷 汪 洋 东为富 ${ }^{*}$ \\ (江南大学化学与材料工程学院, 江苏无锡 214122)
}

\begin{abstract}
摘要: 基于 PDGI/PAAm (PDGI/SN) 层状光子晶体水凝胶, 制备了多重网络 PDGI/PAAm-PAMPS-PAAm $(\mathrm{PDGI} / \mathrm{TN})$ 水凝胶, 将水凝胶浸入 $6: 4(\mathrm{VIV})$ 乙醇水溶液中去溶胀, 记为凝胶 PDGI/TN-0.6。结果表明, 引入 多重网络之后, PDGI/TN 凝胶的溶胀率从 PDGI/SN 的 $850 \% \pm 45 \%$ 提高到 $1070 \% \pm 50 \%$, 颜色从蓝色 $\left(\lambda_{\max }=450 \mathrm{~nm}\right)$ 变为透明, 去溶胀后Bragg 衍射峰为 $670 \mathrm{~nm}$, 实现凝胶的Bragg 衍射峰在 $450-670 \mathrm{~nm}$ 间调 控。拉伸和压缩实验说明, PDGI/TN-0.6力学性能优异, 拉伸强度和压缩强度分别达到 0.99 和 $37.0 \mathrm{MPa}$ 。 同时, PDGI/TN-0.6 具有优异应力变色响应性, 压缩应变 $\left(\varepsilon_{c}\right)$ 从 0 增加到 0.5 , Bragg 衍射峰发生蓝移, 响应 范围覆盖可见光; 当撤去应力后, 立即恢复初始状态, 可重复多次。利用扫描电镜(SEM)进一步研究了层状 光子晶体水凝胶层微观结构。
\end{abstract}

关键词: 层状结构; 光子晶体; 水凝胶; 机械变色

中图分类号: 0648

\section{A Lamellar Photonic Hydrogel Reinforced by Triple Network with Fast and Reversible Mechanochromic Properties}

\author{
XIANG Shuang-Fei LI Ting WANG Yang DONG Wei-Fu* \\ (School of Chemical and Material Engineering, Jiangnan University, Wuxi 214122, Jiangsu Province, P. R. China)
}

\begin{abstract}
The multi-network material PDGI/PAAm-PAMPS-PAAm (PDGI/TN) was synthesized, starting from PDGI/PAAm (PDGI/SN). The PDGI/TN was subsequently deswelled to an equilibrium state in an ethanol/water mixture $(6: 4)(V / V)$ to generate a material termed PDGI/TN-0.6. The results indicate that the swelling radio of the PDGI/TN was increased to $1070 \% \pm 50 \%$, compared with a value of $850 \% \pm 45 \%$ for the original PDGI/SN. In addition, the color of the gel changed from blue $\left(\lambda_{\max }=450 \mathrm{~nm}\right)$ to transparent and the Bragg diffraction peak transitioned to $670 \mathrm{~nm}$. The Bragg diffraction peak could also be tuned over the range from 450 to $670 \mathrm{~nm}$ by adjusting triple network and the ethanol/water solution. Loading-unloading cycling in conjunction with tensile and compressive testing demonstrated that the PDGI/TN-0.6 possessed outstanding mechanical properties. The tensile and compressive stresses of PDGI/TN-0.6 were significantly improved from those of the initial PDGI/ $\mathrm{SN}$, to 0.99 and $37.0 \mathrm{MPa}$, respectively. Loading-unloading cycling data showed that the PDGI/TN-0.6 exhibited excellent responsiveness even after repeated trials. As compressive strain $\left(\varepsilon_{c}\right)$ was increased from 0 to 0.5 , the Bragg diffraction peak underwent a blue shift under compression and eventually spanned the entire visible spectrum wavelength range at the same rate that compressive deformation was applied and released. The effect of the triple network on the inner layer distance in this material was also investigated using scanning electron microscopy (SEM).
\end{abstract}

Key Words: Lamellar structure; Photonic hydrogel; Hydrogel; Mechanochromic

Received: March 21, 2016; Revised: May 9, 2016; Published on Web: May 12, 2016.

*Corresponding author. Email: wfdong@jiangnan.edu.cn; Tel: +86-510-85917763.

The project was supported by the National Natural Science Foundation of China (51373070).

国家自然科学基金(51373070)资助项目

(c) Editorial office of Acta Physico-Chimica Sinica 


\section{1 引言}

响应性光子晶体水凝胶是将光子晶体引入到 水凝胶中, 得到能够对可见光产生 Bragg 衍射的材 料 ${ }^{1-3}$, 当光子晶体水凝胶在外界化学或者物理刺 激下 ${ }^{4-11}$, 其体积的变化导致水凝胶中的晶格间距 发生变化, 引起颜色变化, 易于观察, 在感应和 检测等领域具有潜在的应用前景。

在适量十二烷基硫酸钠存在的条件下，可聚 合表面活性剂十二烷基衣康酸甘油酯(DGI)可以在 水中层层自组装形成双分子层，对可见光产生 Bragg 衍射, 呈现出虹彩现象 ${ }^{12}$ 。Haque 等 ${ }^{13}$ 首先成 功地将十二烷基衣康酸甘油酯(DGI)引入到聚丙烯 酰胺(PAAm)水凝胶中, 聚合形成一维层层结构, 制备得到具有机械响应性的 PDGI/PAAm一维层状 光子晶体水凝胶。PDGI/PAAm 对压缩/拉伸具有颜 色的响应, 但响应及回复周期长达 5-30 min。为 了缩短 PDGI/PAAm 的响应及回复时间, Haque 等 ${ }^{14}$ 在 PDGI/PAAm 的基础上, 引入 PAAm 第二网络, 制备得到 PDGI/PAAm ${ }^{2}$ 双网络光子晶体水凝胶, 响 应回复速度有所提高, 但力学性能下降; 后来, Yue 等 ${ }^{15}$ 通过对丙烯酰胺层水解, 制备得到 PDGIh-PAAm, 响应及回复周期明显缩短, 但力学性能 下降明显。

为了制备高强度水凝胶, Kaneko 等 ${ }^{16}$ 在双网络 基础上, 发展了三网络技术( $\mathrm{TN})$ : 以聚 2-丙烯酰 胺-2-甲基丙磺酸(AMPS) 电解质为第一和第三网 络, 聚丙烯酰胺为第二网络, 制备得到 PAMPS/ PAAm/PAMPS 三网络水凝胶，在应变 70\%的情况 下，压缩强度达到 $10 \mathrm{MPa}$ 。Argun 等 ${ }^{17}$ 制备得 $\mathrm{PAAm} / \mathrm{PAAm} / \mathrm{PAAm}$ 三网络水凝胶，压缩强度达到 $19 \mathrm{MPa}$ 。三网络技术是调控凝胶性能的有效手段。

本文基于 PDGI/PAAm (PDGI/SN) 层状光子晶 体水凝胶, 在 PDGI 层与层之间的 PAAm 层, 引入 三网络, 制备得到多重网络光子晶体水凝胶 PDGI/
PAAm-PAMPS-PAAm (PDGI/TN), 并在 $6: 4(V / V)$ 乙醇水溶液中溶胀平衡, 凝胶层间距的调控如示 意图 1 所示。本文主要研究该凝胶的机械强度、力 学响应性、响应及回复性能。

\section{2 实验部分}

\section{1 主要试剂与仪器}

十二烷基衣康酸甘油酯, 根据文献报道, 实 验室自制 ${ }^{12}$; 2-羟基-4'-(2-差欮乙氧基)-2-甲基苯丙酮 (Irgacure2959)，98\%，萨恩化学技术(上海)有限公 司；2-丙烯酰胺-2-甲基丙磺酸，98\%，上海晶纯生 化科技股份有限公司; 丙烯酰胺(AAm), 分析纯, 国药集团化学试剂有限公司; $N, N^{\prime}$-亚甲基双丙烯 酰胺(MBAA)，99\%，上海晶纯生化科技股份有限 公司; 十二烷基硫酸钠(SDS), $95 \%$, 国药集团化 学试剂有限公司。

紫外灯 (365 nm, $175 \mathrm{~W})$, 苏州锐特玻璃机械 有限公司。光纤光谱仪(FLA5000), 杭州晶飞科技 有限公司; 数码相机(DSC-HX300), 日本 SONY 公司。

\subsection{PDGI/PAAm 层状光子晶体水凝胶的制备}

将得到的 DGI 根据其双亲性的特点, 与水凝 胶单体制成层状光子凝胶, 具体过程如下: 在水 溶液中分别加入 DGI、AAm、交联剂 $N, N^{\prime}$-亚甲基 双丙烯酰胺(MBAA)、光引发剂 Irgacure2959, 浓 度分别是： $\mathrm{DGI}$ 为 $0.1 \mathrm{~mol} \cdot \mathrm{L}^{-1}, \mathrm{AAm}$ 为 $2 \mathrm{~mol}$. $\mathrm{L}^{-1}, \mathrm{MBAA}$ 为 $1 \mathrm{mmol} \cdot \mathrm{L}^{-1}$, Irgacure2959 为 2 $\mathrm{mmol} \cdot \mathrm{L}^{-1}$, 同时加入 $0.01 \mathrm{~g} \cdot \mathrm{L}^{-1}$ 的 SDS, 在 $55^{\circ} \mathrm{C}$ 下超声 $30 \mathrm{~min}$ 至 DGI 形成稳定的双分子层, 出现 虹彩现象, $55^{\circ} \mathrm{C}$ 恒温备用。采用内径为 $0.25 \mathrm{~mm}$ 的注射器将溶液以 $5 \mathrm{~cm} \cdot \mathrm{s}^{-1}$ 速率注入自制水凝胶模 具中, 模具尺寸： $50 \mathrm{~mm} \times 80 \mathrm{~mm} \times(0.5-2 \mathrm{~mm})$, 密封后, 在 $50{ }^{\circ} \mathrm{C}$ 下采用 $175 \mathrm{~W}$ 紫外灯进行光引发 聚合 $3 \mathrm{~h}$ 。

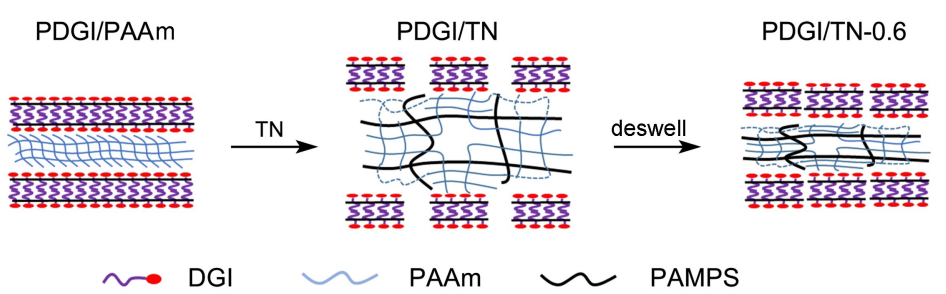

示意图 1 层状结构的调控

Scheme 1 Control of lamellar structure

DGI: dodecyl glyceryl itaconate; PDGI: poly(dodecyl glyceryl itaconate); PAAm: polyacrylamide; PAMPS: poly(2-acrylamido-2-methylpropanesulfonic acid); TN: triple network 


\subsection{PDGI/TN-0.6 层状光子晶体凝胶的制备}

将 PDGI/PAAm 浸入含有 $1 \mathrm{~mol} \cdot \mathrm{L}^{-1}$ 的 AMPS、 $0.04 \mathrm{~mol} \cdot \mathrm{L}^{-1}$ 的 MBAA 以及 $5 \mathrm{mmol} \cdot \mathrm{L}^{-1}$ 的 Irgacure2959 水溶液中溶胀平衡 $7 \mathrm{~d}$, 然后用吸水纸吸 干表面的溶液, 放入模具密闭, 采用功率为 $400 \mathrm{~W}$ 紫外灯光照 $1 \mathrm{~h}$, 制备得到 PDGI/PAAm-PAMPS 层 状光子晶体水凝胶。再将 PDGI/PAAm-PAMPS 浸 泡在含有 $2 \mathrm{~mol} \cdot \mathrm{L}^{-1}$ 的 $\mathrm{AAM} 、 1 \mathrm{mmol} \cdot \mathrm{L}^{-1}$ 的 $\mathrm{MBAA}$ 及 $2 \mathrm{mmol} \cdot \mathrm{L}^{-1}$ 的 Irgacure2959 水溶液中溶胀平衡 7 d。采用同样的方法，去除表面的溶液，之后采用 功率为 $400 \mathrm{~W}$ 紫外灯下 $1 \mathrm{~h}$, 制备得到 PDGI/ PAAm-PAMPS-PAAm (PDGI/TN), 放入水中溶胀 平衡, 去除残留单体。

将制备得到的 PDGI/TN 层状光子晶体水凝 胶, 截取所需形状和大小, 放入 $6: 4(V / V)$ 乙醇水 溶液中, 溶胀平衡 $36 \mathrm{~h}$, 即得到 PDGI/TN-0.6 层状 光子晶体凝胶。

\section{4 溶胀/去溶胀}

溶胀/去溶胀在本文中主要用于调节凝胶的层 间距。首先用直径为 $10 \mathrm{~mm}$ 的裁刀将凝胶裁成圆 柱型。随后将其置于干燥箱里烘干至恒重, 再在 去离子水中进行溶胀, 隔一定时间将其取出, 用 滤纸擦干表面水滴, 进行称量。去溶胀过程是将 在水中完全溶胀之后的凝胶, 用滤纸擦干表面水 滴, 在 $6: 4(V / V)$ 乙醇水溶液中进行, 去溶胀至恒 重, 进行称量。溶胀/去溶胀的平衡溶胀率(SR)由公 式 $\mathrm{SR}=\left(M_{\mathrm{s}}-M_{\mathrm{d}}\right) / M_{\mathrm{d}} \times 100 \%$ 计算而得，其中 $M_{\mathrm{s}}$ 为 平衡溶胀/去溶胀的重量, $M_{\mathrm{d}}$ 为相应干凝胶的重量。

\subsection{Bragg 衍射光谱分析}

采用FLA5000 微型光纤光谱仪, 对材料进行 反射光谱的测定。具体的方法是取所要测试的光 子凝胶, 放置于黑色底板上, 将其表面的水用滤 纸吸干, 将探头放置在光子凝胶表面, 与待测光 子水凝胶表面呈 $90^{\circ}$, 进行测试, 得到 Bragg 衍射 波谱图。

\section{6 拉伸性能测试}

在室温条件下，采用万能测试机对其进行拉 伸性能测试(试样尺寸 $50 \mathrm{~mm} \times 10 \mathrm{~mm} \times(2-3) \mathrm{mm}$ 哑铃型, 标距长度为 $15 \mathrm{~mm}$, 内部宽度为 $4 \mathrm{~mm}$, 拉伸速率为 $20 \mathrm{~mm} \cdot \mathrm{min}^{-1}$ 。记录实验测得的试样拉 伸应变 $\left(\varepsilon_{t}\right)$ 及对应的拉伸应力 $\left(\sigma_{t}\right)$ 。

\section{7 压缩性能测试}

采用万能测试机对样品进行压缩力学性能测
试(试样尺寸直径 6-8 $\mathrm{mm}$ 圆柱型), 压缩速度为 $2 \mathrm{~mm} \cdot \mathrm{min}^{-1}$ 。记录实验测得的试样压缩应变 $\left(\varepsilon_{\mathrm{c}}\right)$ 及 对应的压缩应力 $\left(\sigma_{c}\right)$ 。

\section{8 扫描电镜}

采用场发射扫描电子显微镜(日立 S-4800, 加 速电压 $10 \mathrm{kV}$ )对其微观形貌进行研究。将样品冷冻 干燥后, 在液氮中脆断, 喷金, 然后通过 SEM 对 其断裂面进行观察。

\section{3 结果与讨论}

\section{1 溶胀性能}

图 1 为 PDGI/SN 和 PDGI/TN 水凝胶的溶胀曲 线图。从图 1 中可以看出, 随着时间的增加, 溶胀 率逐渐增大，样品均在溶胀 $35 \mathrm{~h}$ 之后达到溶胀平 衡。 PDGI/SN 的水凝胶的平衡溶胀率为 $850 \% \pm$ $45 \%$, 当引入 PAMPS 阴离子网络后, PDGI/TN 水 凝胶的平衡溶胀率明显增加, 达到 $1070 \% \pm 50 \%$ 。 图 $2 \mathrm{~A}$ 为 $\mathrm{PDGI} / \mathrm{SN}$ 的数码照片, 呈现蓝色, 测得 Bragg 衍射峰 $\lambda_{\text {max }}=450 \mathrm{~nm}$ 。当引入三重网络后, 凝胶呈透明状，如图 2B 所示。这是由于三重网络 的作用使得层间距增大造成的 ${ }^{11}$ 。将凝胶 PDGI/TN 放入 $6: 4(V / V)$ 乙醇水溶液中去溶胀, 由于 PDGI/ $\mathrm{TN}$ 中水的渗透压高于乙醇溶液，PDGI/TN 在乙醇

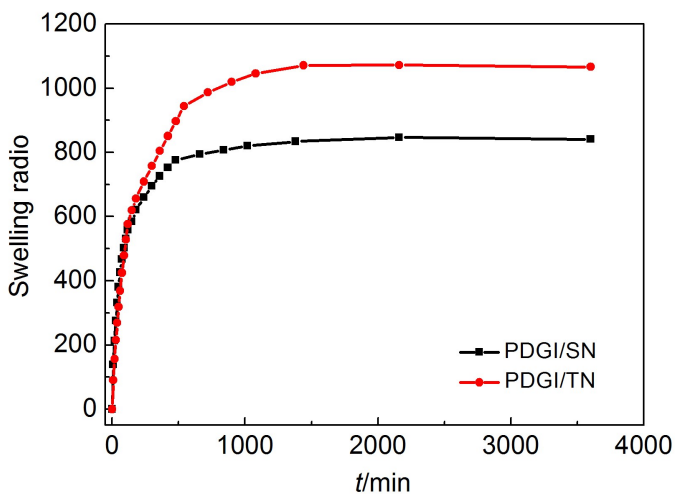

图 $1 \mathrm{PDGI} / \mathrm{SN}$ 和 PDGI/TN 的溶胀曲线

Fig.1 Swelling curves of PDGI/SN and PDGI/TN
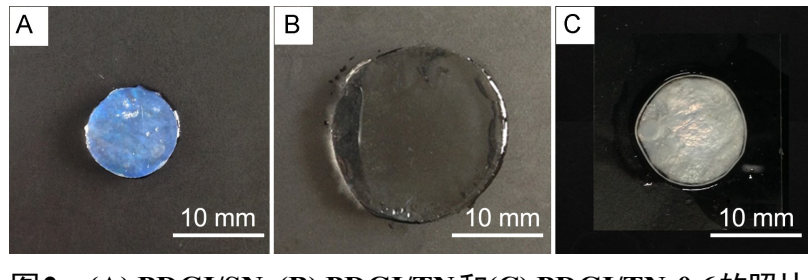

图 2 (A) PDGI/SN, (B) PDGI/TN 和(C) PDGI/TN-0.6 的照片

Fig.2 Photographs of (A) PDGI/SN, (B) PDGI/TN, and

(C) PDGI/TN-0.6 

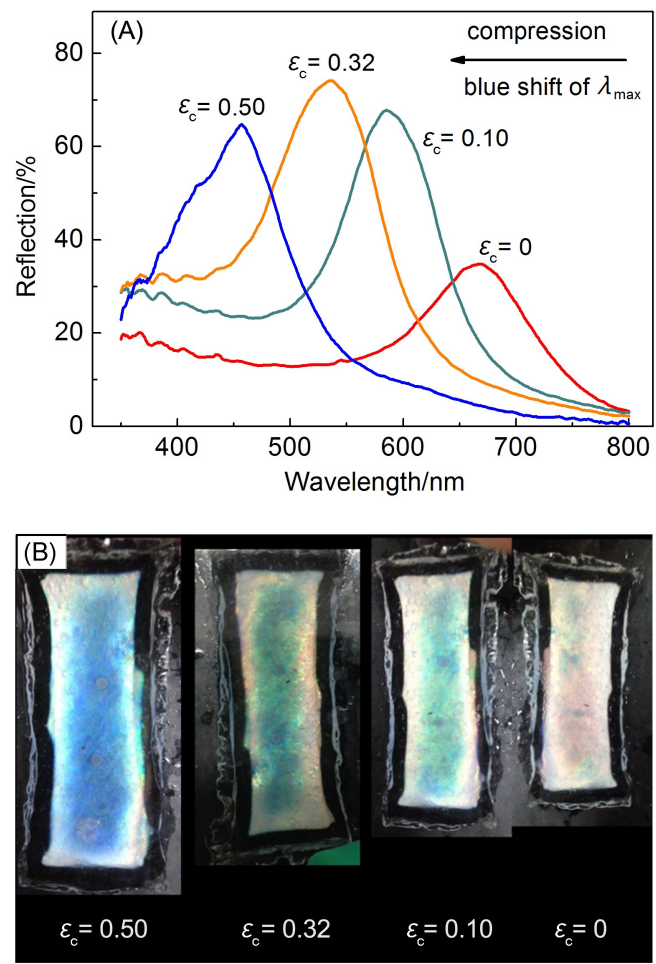

图 3 (A) PDGI/TN-0.6 在压缩过程中的 Bragg 衍射光谱 图; (B)压缩过程中凝胶的数码照片

Fig.3 (A) Bragg diffraction spectra of PDGI/TN-0.6 under compression and (B) photographs of hydrogel under compression $\varepsilon_{c}:$ compressive strain

水溶液中失水, 当渗透压平衡后, 溶胀率为 $19.8 \% \pm 1.0 \%, B r a g g$ 衍射峰 $\lambda_{\text {max }}=670 \mathrm{~nm}$, 呈现红 色。在互穿网络和乙醇水溶液的共同作用下，层 状光子凝胶的 Bragg 衍射波长可控, 可覆盖可见光 区域，与文献 ${ }^{18,19}$ 报道相似。

\subsection{PDGI/TN-0.6 对压应力的响应}

图 3 是 PDGI/TN-0.6 在压缩过程中的衍射光谱 图, 可以看出 PDGI/TN-0.6 光子水凝胶的压缩应变 $\left(\varepsilon_{\mathrm{c}}\right)$ 在 $0-0.5$ 范围变化时, 在可见光范围内对压缩 应力具有颜色响应变化, 衍射波长发生明显蓝 移。PDGI/TN-0.6 光子晶体水凝胶在未施加压缩应

表 1 PDGI/SN 和PDGI/TN-0.6 的拉伸性能 Table 1 Tensile properties of PDGI/SN and PDGI/TN-0.6

\begin{tabular}{ccc}
\hline Sample & $\sigma_{/} / \mathrm{kPa}$ & $\varepsilon_{/}\left(\mathrm{mm} \cdot \mathrm{mm}^{-1}\right)$ \\
\hline PDGI/SN & $74 \pm 2$ & $11.98 \pm 0.79$ \\
PDGI/SN-0.6 & $231 \pm 24$ & $9.66 \pm 0.72$ \\
PDGI/TN & $405 \pm 17$ & $7.12 \pm 0.43$ \\
PDGI/TN-0.6 & $990 \pm 11$ & $6.37 \pm 0.80$ \\
\hline
\end{tabular}

$\sigma_{\mathrm{t}}$ and $\varepsilon_{\mathrm{t}}$ indicate the tensile fracture stress and fracture strain of hydrogel.

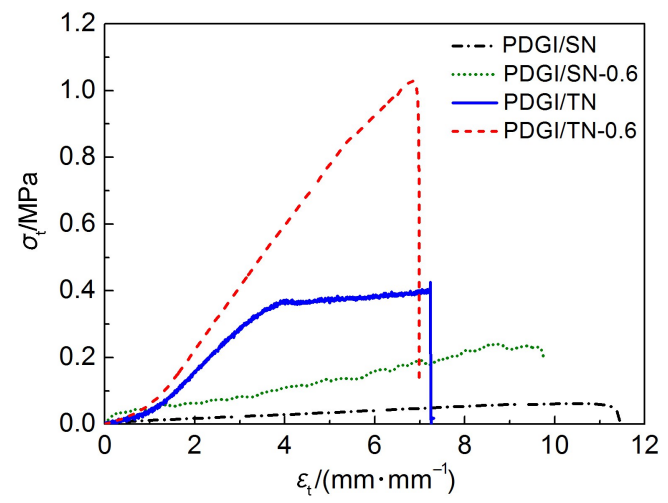

图 $4 \mathrm{PDGI} / \mathrm{SN}, \mathrm{PDGI} / \mathrm{SN}-0.6, \mathrm{PDGI} / \mathrm{TN}$ 和 PDGI/TN-0.6 的拉伸应力 - 应变图

Fig.4 Tensile stress - strain curves of PDGI/SN, PDGI/SN-0.6, PDGI/TN and PDGI/TN-0.6

力时 $\varepsilon_{\mathrm{c}}=0, \lambda_{\text {max }}=670 \mathrm{~nm}$ (红色)。当对其施加连续 的压缩应力, 压缩应变从 $\varepsilon_{\mathrm{c}}=0$ 增加到 $\varepsilon_{\mathrm{c}}=0.5$ 的过 程中, PDGI/TN-0.6 做出连续响应, 当 $\varepsilon_{\mathrm{c}}=0.5$ 时, $\lambda_{\text {max }}=460 \mathrm{~nm}$ (蓝色)。这是由于在压缩过程中，凝 胶的层间距缩小, Bragg 衍射峰波长减小, 可见光 波长向低波长移动，在压缩过程实现了颜色响 应。该响应范围为 460-670 nm，基本覆盖了可见 光区域。

\section{3 拉伸性能}

表 1 列出了 PDGI/SN, PDGI/SN-0.6, PDGI/ $\mathrm{TN}$ 和 PDGI/TN-0.6 光子水凝胶的拉伸性能数据。 图 4 为 $\mathrm{PDGI} / \mathrm{SN}, \mathrm{PDGI} / \mathrm{SN}-0.6, \mathrm{PDGI} / \mathrm{TN}$ 和 $\mathrm{PDGI} /$ $\mathrm{TN}-0.6$ 光子水凝胶的拉伸应力 - 应变曲线。从表 1 和图 4 中可见, $\mathrm{PDGI} / \mathrm{SN}$ 光子凝胶的拉伸强度为 $(74 \pm 2) \mathrm{kPa}$, 断裂伸长率为 $1198 \%$ 。而 PDGI/TN0.6 光子水凝胶的拉伸强度为 $(990 \pm 11) \mathrm{kPa}$, 比 $\mathrm{PDGI} / \mathrm{SN}$ 提高了 12 倍，但断裂伸长率降为 $637 \%$ 。 可见，引入第三网络及在乙醇水溶液中去溶胀可 使水凝胶的力学性能明显提高。

\section{4 压缩性能}

表 2 列出了 $\mathrm{PDGI} / \mathrm{SN}$ 和 PDGI/TN-0.6 光子水凝

表 2 PDGI/SN 和 PDGI/TN-0.6 的压缩性能

Table 2 Compression properties of PDGI/SN and PDGI/TN-0.6

\begin{tabular}{cccc}
\hline Sample & $\sigma_{\mathrm{c}, 0.9} / \mathrm{MPa}$ & $\sigma_{\text {fracture }} / \mathrm{MPa}$ & $\varepsilon_{\text {fracture }} /\left(\mathrm{mm} \cdot \mathrm{mm}^{-1}\right)$ \\
\hline PDGI/SN & $0.69 \pm 0.2$ & $2.56 \pm 0.3$ & 0.99 \\
PDGI/TN-0.6 & $24.7 \pm 1.9$ & $37.0 \pm 2.3$ & 0.99 (no fracture) \\
\hline
\end{tabular}

$\sigma_{\mathrm{c}, 0.9}$ indicates the compressive stress of the hydrogel at a strain of $90 \%$. $\sigma_{\text {fracture }}$ and $\varepsilon_{\text {fracture }}$ indicate fracture compressive stress and strain, respectively. 


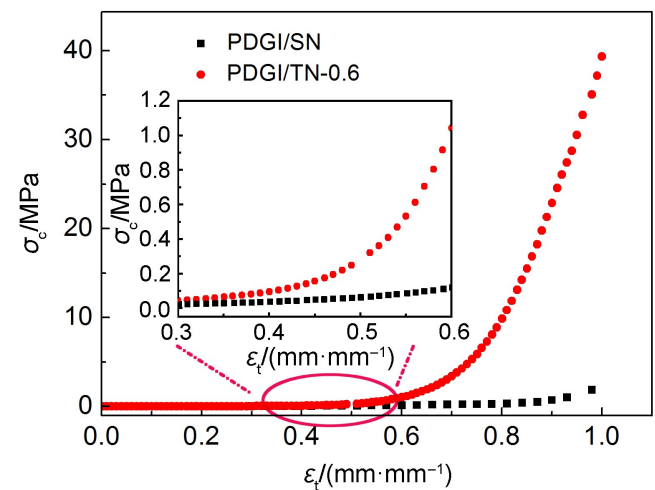

图 $5 \mathrm{PDGI} / \mathrm{SN}$ 和 PDGI/TN-0.6 的压缩应力-应变曲线

Fig.5 Compressive stress-strain curves of PDGI/SN and PDGI/TN-0.6

胶的压缩性能, 图 5 为 PDGI/SN 和 PDGI/TN-0.6 光 子水凝胶的压缩应力 - 应变曲线。从表 2 和图 5 中 可见, 压缩应变为 0.99 时, PDGI/SN 光子凝胶的 压缩强度为 $2.56 \mathrm{MPa}$, 而 PDGI/TN-0.6 光子凝胶的 压缩强度为 $37.0 \mathrm{MPa}$, 提高了 14 倍, 并且未发生 破裂, 可见三重网络光子凝胶比单网络光子凝胶 的压缩性能明显提高。这是由于凝胶中引入 PAMPS 刚性网络作为支撑, 提高其压缩强度, 而 PAAm 作为柔性网络耗散能量, 提高了 PDGI/TN0.6 光子水凝胶的压缩强度。

\section{5 重复应力变色}

单网络水凝胶虽然能够对压缩应变进行瞬时 响应, 然而响应后, 无法立即恢复到初始状态, 恢复响应的时间大于 $10 \mathrm{~min}^{11}$ 。对本研究的 PDGI/ $\mathrm{TN}-0.6$ 施加压缩应力, 在 $\varepsilon_{\mathrm{c}}=0-0.5$ 范围内, 它能 立即做出响应, 响应范围覆盖 460-670 nm, 撤去 压应力, 能瞬时恢复到初始的反射波长, 且具有

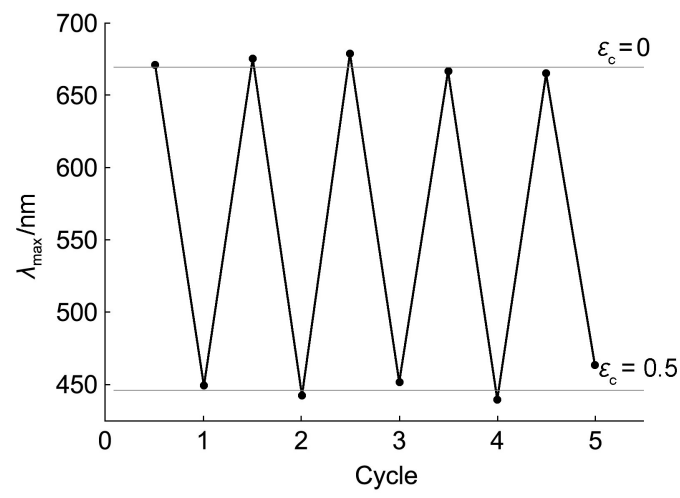

图 6 重复加载-卸载压应力过程中 PDGI/TN-0.6 在 压应变 $\varepsilon_{\mathrm{c}}=0$ 和 $\varepsilon_{\mathrm{c}}=0.5$ 时的 Bragg 衍射峰

Fig.6 Repeat of loading-unloading cycles of PDGI/TN-0.6 and Bragg diffraction peaks of PDGI/TN-0.6 at compress strain $\varepsilon_{\mathrm{c}}=0$ and $\varepsilon_{\mathrm{c}}=0.5$
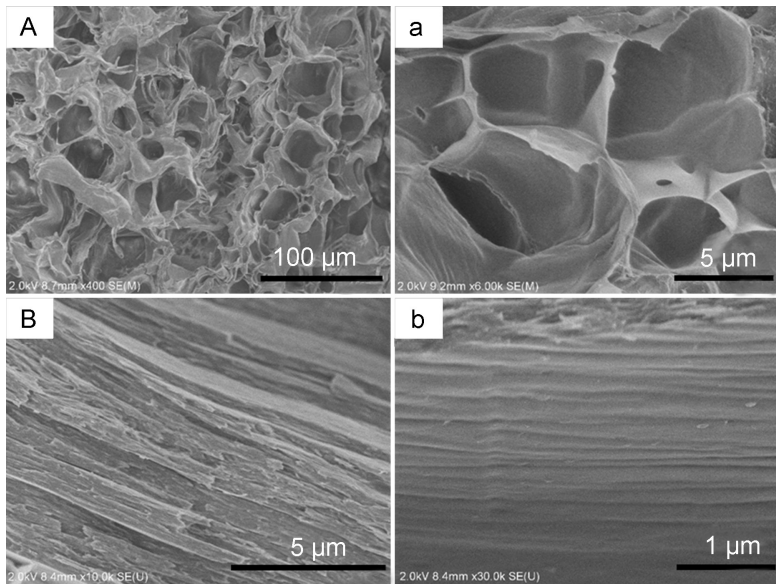

图 7 PAAm $(A, a)$ 和 PDGI/SN $(B, b)$ 的扫描电镜图

Fig.7 Scanning electron microscopy (SEM) images of

PAAm (A, a) and PDGI/SN (B, b)

优异的可重复性, 重复 5 次以上, 仍然具有很好的 响应性, 如图6所示。这是由于在三网络的基础 上, PDGI/SN 光子水凝胶中引入 PAMPS 网络, 由 于 PAMPS 吸水性强, 扩大了 PDGI/SN 中的 PAAm 凝胶层, 同时填入 PAAm 为中性网络, 这就极大地 增加了光子水凝胶的强度和韧性, 因此, 当撤去 外力后, 凝胶可瞬时恢复到初始状态。

\section{6 扫描电镜(SEM)}

图 7(A, a) 为纯丙烯酰胺水凝胶的 SEM 图, 将 稳定后的 DGI 前驱液通过注射的方式, 注入模 具, 紫外光引发聚合, DGI 在注射过程中，沿着注 射方向进行取向，同时改变了聚丙烯酰胺水凝胶 的微观结构, 形成层层结构光子水凝胶。图 7(B, b)组图为 PDGI/PAAm 水凝胶的 SEM 图, 宏观上呈 现蓝色 $\left(\lambda_{\text {max }}=460 \mathrm{~nm}\right)$, 图中的层间距约为 $d=$ 100-200 nm。由此说明成功地制备了 PDGI/SN 光 子晶体水凝胶。

\section{4 结 论}

采用 PDGI/PAAm (蓝色, $\lambda_{\text {max }}=450 \mathrm{~nm}$ ), 通过 三重网络技术, 制备得到 PDGI/PAAm- PAMPSPAAm (PDGI/TN) 层状光子晶体水凝胶, 在体积比 为 $6: 4$ 乙醇水中去溶胀后, 得到 PDGI/TN-0.6 (红 色, $\lambda_{\text {max }}=670 \mathrm{~nm}$ ), 通过调节水凝胶的体积实现对 层状光子晶体水凝胶 Bragg 衍射波长的调控。

与 $\mathrm{PDGI} / \mathrm{SN}$ 单网络水凝胶相比, PDGI/TN-0.6 的力学性能大幅提高, 拉伸强度和压缩强度分别 由 $74 \mathrm{kPa}$ 和 $2.56 \mathrm{MPa}$ 提高到 0.99 和 $37.0 \mathrm{MPa}$ 。并 且 PDGI/TN-0.6 能够对压缩应变 $\varepsilon_{\mathrm{c}}=0-0.5$ 做出响 
应, 响应范围覆盖 460-670 nm, 经过重复压缩-颜 色响应测试, PDGI/TN-0.6 能够对压力立即做出响 应, 撤去应力, 立即恢复, 具有优异的压力变色 响应性。

\section{References}

(1) Hayakawa, M.; Onda, T.;Tanaka, T. Langmuir 1997, 13 (14), 3595. doi: 10.1021/1a970195x

(2) Lee, K.; Asher, S. A. J. Am. Chem. Soc. 2000, 122 (39), 9534. doi: $10.1021 / \mathrm{ja} 002017 \mathrm{n}$

(3) Tsuji, S.; Kawaguchi, H. Langmuir 2005, 21 (18), 8439. doi: 10.1021/la050271t

(4) Lim, H. S.; Lee, J. H.; Walish, J. J. ACS Nano 2012, 6 (10), 8933. doi: $10.1021 / \mathrm{nn} 302949 \mathrm{n}$

(5) Yue, Y. F.; Haque, M. A.; Kurokawa, T. Adv. Mater. 2013, 25 (22), 3106. doi:10.1002/adma.201300775

(6) Baek, Y. B. Mol. Cryst. Liq. Cryst. 2014, 600 (1), 9. doi: 10.1080/15421406.2014.936341

(7) Xu, X. L.; Friedman, G.; Humfeld, K. D. Chem. Mater. 2002, 14 (3), 1249. doi: $10.1021 / \mathrm{cm} 010811 \mathrm{~h}$

(8) MacConaghy, K. I.; Geary, C. I.; Kaar, J. L. J. Am. Chem. Soc. 2014, 136 (19), 6896. doi: 10.1021/ja5031062

(9) Foulger, S. H.; Ping, J.; Lattam, A. C. Langmuir 2001, 17 (19),
6023. doi: $10.1021 / 1 \mathrm{a} 010264 \mathrm{e}$

(10) Chen, M.; Guan, Y.; Zhang, Y. J. Angew. Chem. Int. Edit. 2013, 52 (38), 10145. doi: 10.1002/ange.201302466

(11) Chen, M.; Guan, Y.; Zhang, Y. J. Angew. Chem. Int. Edit. 2015, 54 (32), 9257. doi: 10.1002/ange.201503004

(12) Naitoh, K.; Ishii, Y.; Tsujii, K. J. Phys. Chem. 1991, 95 (20), 7915. doi: 10.1021/j100173a066

(13) Haque, M. A.; Kamita, G.; Kurokawa, T.; Tsujii, K.; Gong, J. P. Adv. Mater. 2010, 22, 5110. doi: 10.1002/adma.201002509

(14) Haque, M. A.; Kurokawa, T.; Kamita, G. Chem. Mater. 2011, 23 (23), 5200. doi: $10.1021 / \mathrm{cm} 2021142$

(15) Yue, Y. F.; Kurokawa, T.; Haque, M. A. Nat. Commun. 2014, 5 (5), 4659. doi: 10.1038/ncomms5659

(16) Kaneko, D.; Gong, J. P.; Osada, Y. Adv. Mater. 2005, 17 (5), 535. doi: 10.1002/adma.200400739

(17) Argun, A.; Can, V.; Okay, O. Macromolecules 2014, 47 (18), 6430. doi: $10.1021 / \mathrm{ma} 5014176$

(18) Liu, S. R.; Yang, Z. K.; Chen, M. Q. Acta Polym. Sin. 2015, No. 8, 957. [刘士荣, 杨兆昆, 陈明清. 高分子学报, 2015, No. 8, 957]

(19) Dong, J.; Cao, Y. X.; Qian, W. P. Acta Phys. -Chim. Sin. 2006, 22 (9), 1090. [董 健, 曹跃霞, 钱卫平. 物理化学学报, 2006, 22 (9), 1090.] doi: 10.3866/PKU.WHXB20060911 